Case Report

\title{
Diagnóstico e tratamento de acidente ofídico por serpente do gênero Bothrops em cão
}

Received: dec, 2019; Accepted: may, 2020

\author{
Leila Maria de Carvalho Alves ${ }^{1}$, Juliana de Abreu Pereira $2^{2}$, \\ Carla Letícia Medeiros Silva Barreto ${ }^{2}$. Marcelo Augusto Silva Barreto ${ }^{2}$, \\ Katherinne Barth Wanis Figueiredo, Natascha Jenifer Morante ${ }^{2}$
}

\begin{abstract}
Resumo
Refere-se como acidente ofídico todo quadro de envenenamento decorrente da inoculação de veneno, através de presas de serpentes. $\mathrm{O}$ veneno das espécies do gênero Bothrops, ao ser inoculado no animal, causa sinais clínicos graves que, se não tratados o mais rápido possível, podem levar o animal ao óbito. O gênero Bothrops (jararacas) está distribuído por todo o território nacional sendo responsável pela maioria dos acidentes ofídicos no país. Diante disso, o objetivo do presente relato foi descrever o diagnóstico e o tratamento hospitalar bem sucedido de acidente botrópico em cão.
\end{abstract}

Palavras-chave: intoxicação, serpente, animais domésticos.

\section{Introdução}

Refere-se como acidente ofídico ou ofidismo todo quadro de envenenamento decorrente da inoculação de veneno, através de presas de serpentes, podendo estas serem de diversos gêneros. Dentre os mais comuns encontram-se os gêneros Crotalus, Bothrops, Lachesis e Micrurus (BRASIL, 2017). O gênero Bothrops (jararacas) está distribuído por todo o território nacional sendo responsável pela maioria dos acidentes ofídicos

\footnotetext{
${ }^{1}$ Programa de Pós-Graduação em Medicina Veterinária da Universidade Federal Rural do Rio de Janeiro (PPGMV - UFRRJ).

${ }^{2}$ Curso de Medicina Veterinária da Universidade Sociedade Educacional de Santa Catarina (Unisociesc), campus Joinville.
}

* Corresponding author: juliana.abreu@unisociesc.com.br, Universidade Sociedade Educacional de Santa Catarina. Rua Albano Schmidt, 3333 - Boa Vista - Joinville, SC - Brasil. CEP: 89206-001. Telefone: (47) 3512-3100 
no país (CHERUBINI et al., 2000; NASCIMENTO, 2000; FRANCO et al., 2001; NOGUEIRA, 2011).

O veneno das espécies do gênero Bothrops, ao ser inoculado no animal, causa sinais clínicos como dor intensa, edema, eritrema, petéquias, hemorragia, hematúria, epistaxe, hematêmese, hipotensão e choque. Isso se dá principalmente devido à ação do veneno botrópico, pois este apresenta propriedades proteolíticas, necrosantes, anticoagulantes, vasculotóxicas, nefrotóxicas, miotóxicas e neurotóxicas (NOGUEIRA, 2011, BERNARDE, 2014; BLANCO, MELO, 2014; SILVA et al., 2018)

Devido a importância desse tema, em virtude das poucas notificações de ocorrências e da baixa rotina de atendimentos em áreas urbanas, o objetivo do presente relato foi descrever o diagnóstico e o tratamento hospitalar bem sucedido de acidente botrópico em cão.

\section{Relato do caso}

Foi atendido um cão, macho, sem raça definida, com idade de 8 anos e com peso de $9,100 \mathrm{Kg}$; em clínica veterinária particular localizada na região metropolitana do Rio de Janeiro, onde os tutores relataram que o paciente havia sido picado na face por uma serpente. $O$ animal peçonhento foi visualizado e morto pelos responsáveis pelo animal. O cão apresentava como único sinal clínico um severo edema na face. Imagens da serpente permitiram ao médico-veterinário responsável identificar o animal como sendo do gênero Bothrops (Figura 01), confirmando a suspeita clínica inicial de acidente botrópico. 
Figura 01: Imagens do animal peçonhento causador do acidente. O padrão de coloração das escamas podendo variar entre cinza, verde, pardo, marrom e amarelo; com escama apresentando um desenho em "V" e região ventral de coloração clara e única permitiu a identificação da serpente como pertencente ao gênero Bothrops.
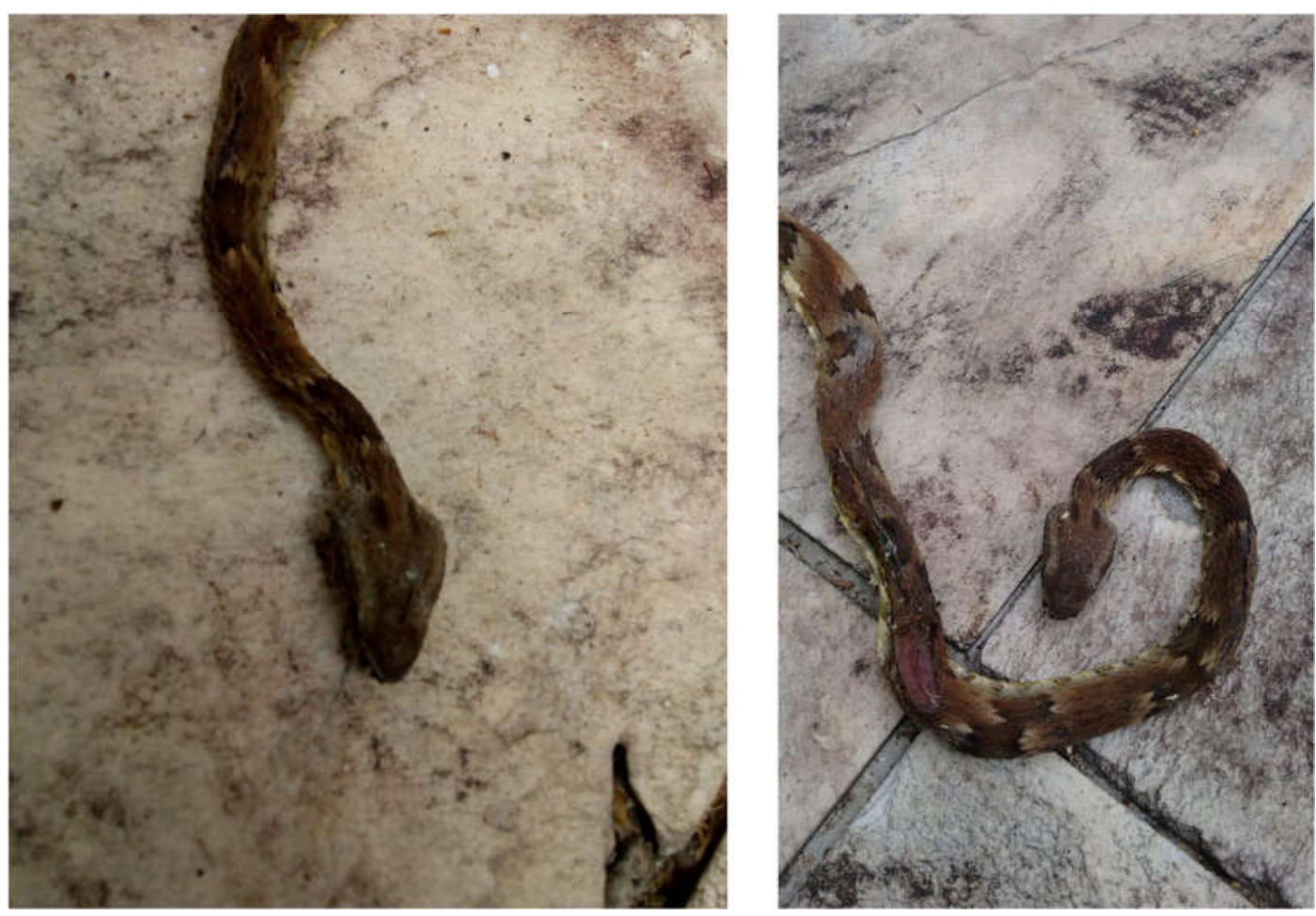

Prontamente o paciente foi submetido a atendimento hospitalar, com implementação de fluidoterapia utilizando solução fisiológica $\mathrm{NaCl}$ 0,9\% na taxa de infusão de 7,5 ml/hora; e administração de soro antiofídico polivalente veterinário (Master Soro Plus, Vencofarma) totalizando o volume administrado de $20 \mathrm{ml}$, por via subcutânea, conforme recomendação do fabricante; objetivando a completa neutralização do veneno e a interrupção das injúrias orgânicas causadas por este.

Para controle das manifestações clínicas associadas aos processos infecciosos e inflamatórios oriundos da picada em si, bem como de possíveis efeitos sobre o trato gastrointestinal, foram administrados os seguintes fármacos: Dexametasona na dose de $1 \mathrm{mg} / \mathrm{kg}$, em dose única, por via subcutânea, no momento do atendimento emergencial; Metronidazol na dose 
de $15 \mathrm{mg} / \mathrm{kg}$, a cada 12 horas, por via endovenosa; Ceftriaxona na dose de 50 $\mathrm{mg} / \mathrm{kg}$, a cada 12 horas, por via endovenosa; soro antitetânico (Vencosat soro antitetânico liofilizado veterinário, Vencofarma) na dose de 5 mil UI/animal, em dose única, por via subcutânea, no momento do atendimento emergencial; Omeprazol na dose de $1 \mathrm{mg} / \mathrm{kg}$, a cada 24 horas, por via endovenosa; e Meloxicam na dose de $0,1 \mathrm{mg} / \mathrm{kg}$, a cada 24 horas, por via subcutânea, a partir do segundo dia de internação.

$\mathrm{Na}$ admissão do paciente foi coletada amostra de sangue para exames de bioquímica sérica. Foi recomendada também a realização do hemograma, porém o tutor não autorizou o exame. Os resultados da análise bioquímica estão representados no Quadro 01.

Quadro 01: Resultados bioquímicos, para avaliação da função hepática, oriundos da amostra obtida no momento da admissão do paciente

\begin{tabular}{|c|c|c|}
\hline Parâmetro & Valor & Referência \\
\hline ALT (TGP) & 52 & $21-102 \mathrm{U} / \mathrm{L}$ \\
\hline AST (TGO) & 146 & $23-66 \mathrm{U} / \mathrm{L}$ \\
\hline Fosfatase alcalina & 597,9 & $20-156 \mathrm{U} / \mathrm{L}$ \\
\hline Ureia & 32 & $15-40 \mathrm{mg} / \mathrm{dL}$ \\
\hline
\end{tabular}

Durante o internamento o paciente foi minuciosamente monitorado, objetivando-se observar possíveis agravamentos do quadro clínico, bem como avaliar a necessidade de administração de nova dose de soro antiofídico. O cão se alimentou normalmente durante todo o período de internação; não apresentou alterações de ritmo e/ou frequência respiratória, nem de coloração de mucosas. Micção e evacuação se encontravam normais, sem alterações no aspecto das excretas. A evolução clínica do paciente quanto à remissão do edema facial pode ser verificada na Figura 02. 
Figura 02: Aspecto físico da face do paciente no momento do acidente, durante o período de internamento; e dois dias após o início do tratamento, evidenciando a completa remissão do edema. Pode-se observar ainda o local onde foi proferida a picada pela serpente.
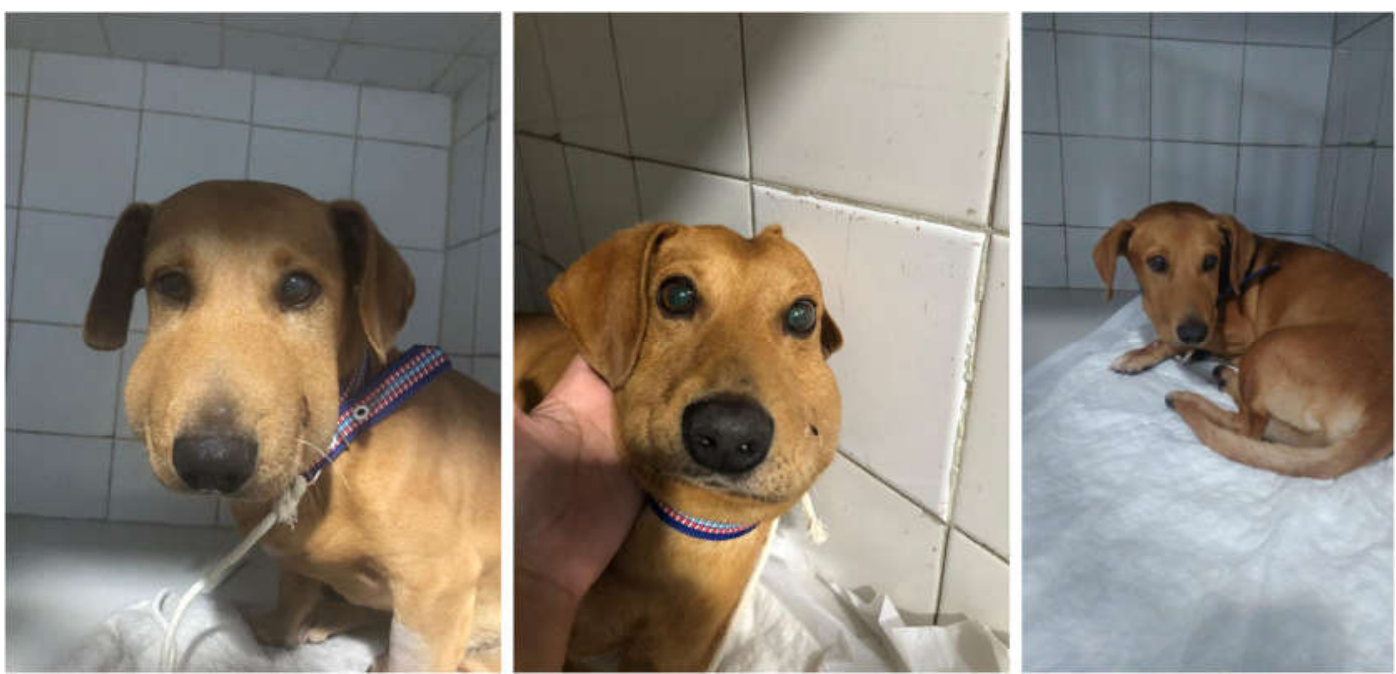

Dois dias após o acidente, o paciente foi liberado para tratamento domiciliar, sendo mantida associação de antibióticos com Espiramicina $25,7 \mathrm{mg} / \mathrm{kg}$ e Metronidazol 13,7 mg/Kg (Stomorgyl 10, Boehringer Inghelheim), a cada 24 horas, por 8 dias; e associação protetora hepática contendo alcachofra, cardo mariano, vitaminas, antioxidantes e aminoácidos (Hepvet, Vetnil), sendo realizada a administração de um comprimido, a cada 24 horas, conforme recomendações do fabricante, pelo período de 30 dias. Foi recomendado ao tutor retornar à clínica imediatamente em caso de agravamento do quadro clínico. Foi marcado retorno para reavaliação clínica posterior um mês após o atendimento. $\mathrm{Na}$ avaliação do retorno, o animal encontrava-se clinicamente sadio e completamente curado do incidente, entretanto os tutores não autorizaram a realização de novos exames laboratoriais 
Alves, Pereira, Barreto, Barreto, Figueiredo, Morante Diagnóstico e tratamento de acidente ofídico...

\section{Discussão}

O maior número de cães acometidos por picada de serpentes ocorre durante as estações mais quentes do ano, como no verão (NOGUEIRA, 2011). Sendo o Rio de Janeiro um estado no qual predomina o clima tropical, essa unidade federativa se torna uma localidade bastante propícia para esse tipo de acidente.

As serpentes do gênero Bothrops possuem fosseta loreal, cauda lisa, desenhos em cores diferentes, variando desde o verde até o preto; e são solenóglifas (JUNIOR, BARRAVIERA, 2001; FONTEQUE, FILHO, SAKATE, 2001). Como no presente caso, tais características permitem a identificação desses animais apenas por meio da visualização de um profissional experiente.

Assim como o animal atendido, os cães são a espécie mais frequentemente envolvida em acidentes ofídicos envolvendo as serpentes do gênero Bothrops (NOGUEIRA, 2011; BARNI et al., 2012; BLANCO, MELO, 2014). A picada normalmente ocorre na região da cabeça e do pescoço do animal, sendo o focinho o local mais acometido (SAKATE, 2002), o que vem de encontro ao ocorrido com o cão deste relato, o qual foi picado pela serpente na região da face, sendo o principal sinal clínico apresentado, o edema nessa região.

Nesses casos, os sinais clínicos são bastante variáveis, assim como a gravidade do quadro depende de alguns fatores, como características da serpente, espécie e tamanho do animal acometido, tempo até o atendimento, local e quantidade de inoculação do veneno (FONTEQUE, FILHO, SAKATE, 2001; JUNIOR et al., 2003). Entretanto, de modo geral, a reação é intensa e imediata, sendo mais comumente encontrados dor e edema como sinais clínicos principais. Porém, dependendo do veneno e tempo de ação deste no organismo do animal, pode-se apresentar aumento da frequência cardíaca e respiratória, dispnéia, inapetência, prostração, cegueira, hemorragias, 
necrose, choque e óbito (BICUDO, 1999; FONTEQUE, FILHO, SAKATE, 2001; JÚNIOR, BARRAVIERA, 2001; JUNIOR et al., 2003).

Assim como o realizado neste caso, o único tratamento disponível, para quadros de picada de serpentes, é a administração de soro antiofídico polivalente, devendo, sempre que possível, utilizar o soro específico de acordo com a serpente que acometeu o animal (BICUDO, 1999; JUNIOR, JUNQUEIRA, 2000; JUNIOR et al., 2003). Além disso, é importante lembrar que o prognóstico do animal também depende do manejo dos demais sinais clínicos apresentados, sendo importante o controle da dor, a hidratação, a prevenção da injúria renal aguda, de hemorragias e infecções (BICUDO, 1999; JUNIOR, JUNQUEIRA, 2000; FONTEQUE, FILHO, SAKATE, 2001; JUNIOR et al., 2003)

Sabe-se que o cão é a terceira espécie mais suscetível ao veneno de cobras desse gênero. Entretanto todas as espécies de animais domésticos são afetadas pelo veneno, porém a sensibilidade e a gravidade do quadro são variáveis entre elas (BLANCO, MELO, 2014). Além disso, o local afetado, a quantidade de veneno inoculada e o tempo decorrido após o acidente possuem grande influência no prognóstico do animal (ANDRADE, 2002). Neste caso, após a picada, o cão foi prontamente submetido ao atendimento hospitalar veterinário, o que resultou em um desfecho positivo e uma completa recuperação do animal.

\section{Conclusão}

A apresentação clínica do quadro de acidente ofídico, assim como o presente relato é bastante específica, e sendo prontamente atendido e bem manejado clinicamente pode haver um bom prognóstico assim como neste caso.

$$
* * *
$$




\title{
Diagnosis and treatment of ophidic accident by Bothrops snake in a dog
}

\begin{abstract}
Ophidic accident refers to any picture of poisoning resulting from the inoculation of poison through snake prey. The venom of the Bothrops species, when inoculated into the animal, causes severe clinical signs that, if not treated as soon as possible, can lead to death. The Bothrops gender (jararacas) is distributed for all national territory as it's responsible for the most ophidic accidents in the country. Therefore, the objective of the present report was to describe the diagnosis and successful hospital treatment of bothropic accident in a dog.

Keywords: poisoning, snake, domestic animals
\end{abstract}

$* * *$

\section{Referências}

ANDRADE S.F Manual de Terapêutica Veterinária, $2^{\circ}$ ed. São Paulo: Roca, p.545$547,2002$.

BARNI, B.S.; MOTTIN, I.B.; VIDOR, S.B.; ALBUQUERQUE, P.B.; CONTESINI, E.A. Incidência e perfil dos animais atendidos devido a acidente ofídico no Hospital de Clínicas Veterinárias da Universidade Federal do Rio Grande do Sul entre os anos de 2005 e 2010. Acta Scientiae Veterinariae, v.40 (Suppl.1), p.1-60, 2012.

BERNARDE, P.S. Serpentes peçonhentas e acidentes ofídicos no Brasil. São Paulo: Anolisbook, p.221, 2014.

BICUDO, P.L. Acidentes ofídicos em Medicina Veterinária. In: BARRAVIERA, B. Venenos: aspectos clínicos e terapêuticos dos acidentes por animais peçonhentos. Rio de Janeiro: EPUB, 1999. p.375-387.

BLANCO, B.S.; MELO, M.M. Acidente botrópico. Cadernos Técnicos de Veterinária e Zootecnia, v.75, p.1-77, 2014. 
Alves, Pereira, Barreto, Barreto, Figueiredo, Morante Diagnóstico e tratamento de acidente ofídico...

BRASIL. Ministério da Saúde. Acidentes por animais peçonhentos - Serpentes. Brasília, DF, 2017. Disponível em: http://portalms.saude.gov.br/saude-de-az/acidentes-por-animais-peconhentos-serpentes. Acesso em: 22 de maio de 2019.

CHERUBINI, A. L.; BICUDO, P. L.; FERREIRA JUNIOR, R. S.; BARRAVIERA, B. Toxicologia veterinária on-line. Revista de Educação Continuada. CRMV-SP, São Paulo, v.3, n.1, p.62-65, 2000. https://doi.org/10.36440/recmvz.v3i1.3354

FONTEQUE, J.H.; FILHO, I.R.B.; SAKATE, M. Acidentes botrópicos de interesse em animais domésticos. Revista de Educação Continuada. CRMV-SP, São Paulo, v.4, n.3, p.102-111, 2001. https://doi.org/10.36440/recmvz.v4i3.3311

FRANCO, R.L; ROCHA, C. C.; JORGE, M. T.; RIBEIRO, L.A. Snakebites in southern Minas Gerais state, Brazil. Journal of Venomous Animals and Toxins including Tropical Diseases, v.7, n.1, p.56-68, 2001. https://doi.org/10.1590/S0104$\underline{79302001000100005}$

JÚNIOR, R. S. F.; BARRAVIERA B. Tissue necrosis after canine bothropic envenoming: a case report. Journal of Venomous Animals and Toxins including Tropical Diseases. v.7, n.2, p.302-312, 2001. https://doi.org/10.1590/S0104$\underline{79302001000200011}$

JUNIOR, R.S.F.; BARRAVIERA, B.; BARRAVIERA, S.R.S., BARRELLA, T.H., VILELA, F.C. Conduta em picadas de serpentes brasileiras em cães e gatos. Revista Científica de Medicina Veterinária - Pequenos Animais e Animais de Estimação, Curitiba, v.1, n.2, p.124-133, 2003.

JUNIOR, R.S.F.; JUNQUEIRA, M.E. Acidentes com animais peçonhentos. Centro de Estudos de Venenos e Animais Peçonhentos - CEVAP/UNESP, 2000. 47p.

NASCIMENTO, S.P. Aspectos epidemiológicos dos acidentes ofídicos ocorridos no Estado de Roraima, Brasil, entre 1992 e 1998. Cadernos de Saúde Pública, v.16, n.1, p.271-276, 2000. https://doi.org/10.1590/S0102-311X2000000100031 
Alves, Pereira, Barreto, Barreto, Figueiredo, Morante Diagnóstico e tratamento de acidente ofídico...

NOGUEIRA, R.M.B. Lagartas e serpentes. In: Nogueira R.M.B \& Andrade S.F. (Eds), Toxicologia Veterinária. Roca, São Paulo, p.323, 2011. https://doi.org/10.1590/S0101-41612011000200005

SAKATE, M. Terapêutica das Intoxicações. In: ANDRADE, S. F. Manual de Terapêutica Veterinária. $2^{\text {a }}$ ed. São Paulo - SP: Roca, 2002, Cap. 21, p. 545 a 547.

SILVA, L.G.; PANZIERA, W.; LESSA, C. A. C.; DRIEMEIER, D. Epidemiological and clinical aspects of ophidian bothropic accidents in dogs. Pesquisa Veterinária Brasileira, v. 38, n. 11, p. 2146-2149, 2018. https://doi.org/10.1590/1678-5150-pvb$\underline{5889}$ 